\title{
Construction of Group Events Risk Field Model Based on the Integrated Framework for Disaster Risk
}

\author{
Kaitai Sun \\ School of Public Administration, Jinan University, Guangzhou, China \\ Email: 375981206@qq.com
}

How to cite this paper: Sun, K.T. (2016) Construction of Group Events Risk Field Model Based on the Integrated Framework for Disaster Risk. Open Journal of Social Sciences, 4, 164-176.

http://dx.doi.org/10.4236/jss.2016.411013

Received: November 2, 2016

Accepted: November 25, 2016

Published: November 28, 2016

Copyright $(\odot 2016$ by author and Scientific Research Publishing Inc. This work is licensed under the Creative Commons Attribution International License (CC BY 4.0).

http://creativecommons.org/licenses/by/4.0/

\begin{abstract}
This paper is mainly based on the integrated framework for disaster risk and risk field theory, building an event risk field model and using this model for group events. Then, the paper describes group events for the concept, analyzes the characteristics and types of mass incidents and constructs the risk field model according to the risk of field theory. Finally, the paper gives a case triggered by taxi transport strike incident and puts forward the mass event control strategy according to the use of the risk field model.
\end{abstract}

\section{Keywords}

Integrated Framework for Disaster, Group Events, Risk Model Field

\section{Introduction}

The 21st Century is an era of both opportunities and challenges. Along with further progress of China's reform and opening up, our country has entered the key period of structural changes, or to say, social transformation. As economic capability and living standard of people have been generally enhanced, a great deal of economic disputes and social conflicts have been intertwined with each other; in addition to easier and faster transmission of various new media like the Internet and that expanding rate and frequency of risk have increased, mass incidents occur frequently in China, which have affected social stability and development and thus aroused widespread concern among the social community. As shown in Table 1, the total number of mass incidents between 2011 and 2014 is still rising. Mass incidents primarily include taxi strikes, demonstrations, doctor-patient conflicts, work and market stoppages, public petitions, clashes between police and the people, environmental pollution, housing demolition 
Table 1. The number of mass incidents in recent years.

\begin{tabular}{ccc}
\hline Years & Quantity (million) & Content \\
\hline 2011 & 13.9 & Doctor-patient conflict \\
2012 & 14.3 & People petition \\
2013 & 16.5 & Environmental pollution \\
2014 & 17.2 & Land Conflict \\
\hline
\end{tabular}

Data sources: the analysis report on typical group events in Chinese society.

and others. To study management of group events is of theoretical and practical significance.

Social risk is an inevitable product of human society's development, and has been intensifying with the development of society. In the 1980s, as a well-known German sociologist, Beck firstly put forward related concept of "risk society", which then became a hot issue in academic research. British scholar Anthony Giddens depicted such a picture: the possibility of nuclear war, ecological disaster, irresistible population explosion, collapse of global economic exchanges and other potential global disasters, which have outlined the disturbing risky prospect of risk society. China cannot extricate from the globalization of risk, either [1]. Xiao Ying believes that the process of China's integrating into globalization and accelerating of economic construction is a process for China to participate in world risk society and shape its own risk society. As a part of world risk society, China's risk society has its unique generative and operational logics, and factors including consistency between industrial society and risk society, economic development model, utilitarian ideology, government capability and interest pursuits have held vital places in risk generation [2]. Chinese scholar Yuan Fang pointed out that the reason behind formation of risks in modern society rests with that people's practices are built on the basis of unreasonable ideas, which can be then mainly divided into four categories: the idea of humans' confrontation with the nature, the idea of inexhaustible resources, the traditional idea of anthropocentrism, the idea of rational worship as well as omnipotence of science and technology [3].

The proposal of concepts of risk society and risk globalization has put forward new requirements for emergency management, but traditional idea of emergency management has been unable to effectively cope with management of emergencies, which however has to take overall management for the process of emergency events. Tong Xing have suggested constructing a comprehensive analytical framework for risks, disasters and crises in this context, deeming that effective resolution of emergencies requires establishing a handling system concerning the entire process of "risk management--disaster (emergency) management--crisis management". Based on the comprehensive risk-disaster-crisis analytical framework [4], Tong Xing et al. put forward the theory of risk field. It is considered that the risk field is composed of risk source, actors involved and resources available in the field, among which actors involved have complicated relations in the field [5].

In the risk society, risk is everywhere, contradictions among social classes occur con- 
stantly, and mass incidents have taken on a rising tendency. On the concept of mass incident, western scholars have carried out studies of mass incidents quite early, but our country didn't initiate research on the topic until the 1980s. Opinions on Actively Preventing and properly Handling Mass Incidents formulated by General Office of the CPC in 2004 provided a relatively official and formal definition about mass incidents: mass incidents refer to group events caused by contradictions among the people, such as group petitioning by the public who consider their interests to be violated and tend to express their will and make requests to related departments and units by means of illegal gathering, containment or other methods, as well as a series of highlight events during the incubation and formation process of the former. Yan Dao Cheng pointed out that many definitions of mass incidents are overshadowed by strong political overtones and bias, and summarized its definition from four perspectives including the nature, main structure, form of expression and cause of mass incident, indicating that mass incidents refer to mass aggregating behaviors caused by contradictions, which occur when stakeholders of same needs find their expression of interests and requirements restricted or prohibited. Such behaviors are open mass behaviors with certain scales, which happen in public, aim at expressing self-interest demands and may exert certain negative impacts on social stability and order [6]. On mass labor conflicts, Liu Bing believes that mass labor conflicts refer to collective action by labors with identical interest demands in certain political, economic and social environment, who ultimately adopt behaviors such as collective disputes, petitions, strikes, work stoppages, sabotage and demonstrations to fight against employers and achieve common goals when their interests are violated or cannot be satisfied [7].

On characteristics of mass incidents, Wang Hong believes that in China, mass incidents are mainly expressed by agonistic behaviors of part of the masses in a variety of forms against the local government, featuring the government's uncontrollability on the events, diversity of forms, complexity of reasons and diversification of participants [8]. However, Wang Dong analyzed characteristics of current mass incidents from another point of view and generalized as follows: the number of mass incidents increases and the scale expands; organizational feature of mass incidents remains obvious with high frequency; confrontation and violence in mass incidents have been evidently strengthened and results have become increasingly severe [9].

Effective classification of mass incidents is conducive to our comprehensive understanding and research on mass incidents, so as to take targeted management methods against different types of mass incidents. According to goal, characteristics and action orientation, $\mathrm{Yu}$ Jianrong classified mass incidents in recent ten years in China into following five types: activist behavior, rights-safeguarding behavior, social venting case, social turmoil, social dispute and organized crime [10]. Jin Jianghao comprehensively analyzed the classification of mass incidents, and then classified it from five aspects: occurring industry and content nature, difference in subject scale, preparation for incidents, form of expression, and occurring region of the incidents [11].

With regard to mass incidents and improvement in management of mass labor con- 
flicts, scholars in our country have carried out in-depth discussion. Du Qiaosheng proposed that improving interest appeal channel and valuing public voice guidance by media are important ways to deal with mass incidents. Teng Xuewei pointed out that, major methods for solving mass incidents include establishing and improving interest expression mechanism, fully guaranteeing people's discourse power, building up and perfect social catharsis mechanism, as well as easing and controlling social discontents. Xiong Xinfa et al. pointed out that in dealing with strikes such as the strike against Honda, enterprises should insist in principles like understanding their workers, communication and negotiation, achieving minimal loss for coping with such cases. Huang Mingzhen believes that the establishment of effective third-party mediation mechanism serves as an important measure to solve mass strikes, and establishment of such a system should lay emphasis on construction of legal system and supporting facilities. Han Jinhua suggested that guaranteeing independence of enterprises and trade unions as well as establishing enterprises' internal appeal mechanism are demanded in order to deal with mass incidents in enterprises [12] [13] [14] [15] [16].

From the perspective of comprehensive risk-disaster-crisis framework, this paper has focused on studying the relationship between all subjects in risk field operation of mass labor conflicts, so as to improve related management strategy. The main structure of this paper is as follows: systematic analysis of the concept of mass labor conflicts, construction of the comprehensive risk-disaster-crisis framework, operational logic of risk field of mass labor conflicts based on the comprehensive risk-disaster framework, optimization strategy for management of mass labor conflicts.

The innovative points of this paper are as follows: first, based on the theory of comprehensive risk-disaster-crisis framework, the paper has classified and oriented related subjects in risk field operation of mass labor conflicts; second, based on studying the theory of comprehensive risk-disaster-crisis framework, the paper has constructed a model for risk field operation of mass labor conflicts; third, based on constructing the model for risk field operation of mass labor conflicts, the paper has analyzed formation mechanism and evolution forms of mass labor conflicts.

\section{Systematic Analysis of the Concept of Mass Labor Conflicts}

\section{The Concept and Types of Mass Incidents}

In China, there is no unified and strict definition of mass incidents. The improper utilization of the concept cannot clarify a definite research object, so we need to put forward a unified definition for mass incidents and mass labor conflicts.

The author defines the mass incidents as group behaviors made by a certain number of participants expressing their demands, which are caused by internal contradictions among the people and will exert certain influences on social stability unity of the people. Mass labor conflicts refer to mass incidents of agonistic behaviors against employers by labors whose goals, expectations and interests largely differ from those rendered by employers and haven't been properly treated, such as collective disputes, petitions, strikes, stoppage, sabotage, demonstrations and others. 
Effective classification of mass incidents is conducive to our comprehensive understanding and study on mass incidents, so that we can take targeted management measures in terms of different types of incidents. In Figure 1, mass incidents can be classified into mass interests-appealing incident, mass venting incident and mass ideaupholding incident according to root causes of incidents. Interests-appealing incident can be classified into mass incident due to labor conflicts, mass incident due to land expropriation and demolition, mass incident due to environmental pollution, such as: collective stoppage by taxi drivers in Nanjing, Tong'an incident in Suzhou and the mass incident in Xiantao, Hubei. This paper mainly studies mass incident due to labor conflicts. Mass venting incident includes mainly two types: firstly, collective venting evolved from mass interests-appealing incident. Initial participants in such incidents have relatively clear interest demands, but due to involvement and intentional venting of many indirect stakeholders, the nature of incident ultimately changes, such as Ruian Incident; the second type is incidents with whole-process collective irrational venting behaviors, resulting from accidental incidents, and Wanzhou Incident was a typical case. Idea-upholding incident is an activity by the public to promote their thoughts and ideas, such as the boycott demonstration by dog lovers against the dog eating festival in Yulin.

\section{Construction of Comprehensive Risk-Disaster-Crisis Framework}

Currently, emergency in our country can be divided into four categories: natural disasters, accidents, public health events, social security incidents. The main representation of social security incident is the mass emergency. The subjects of mass emergency are those mass incidents aroused by contradictions among the people, including labor disputes, urban housing demolition, environmental pollution and others. Management of such mass incidents has naturally become the focus of mass incident management.

\subsection{To Establish a Whole-Process Comprehensive Risk-Disaster-Crisis Framework}

As one of the important functions of modern government, public crisis management refers to emergency or a state of emergency which happens to the sudden and may bring great losses to people's lives and property safety and affect social stability. Therefore, public crisis is of urgency, criticality, abruptness and highly uncertainty, and will produce" ripple effect". Now, the idea of public crisis management in China hasn't

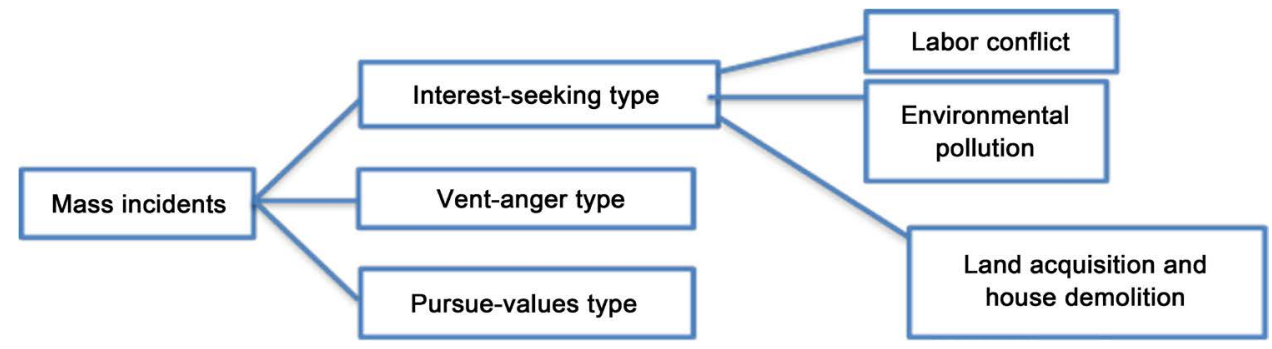

Figure 1. Types of group events. 
completely got rid of the misunderstanding of "emphasize resolution but underestimate management", ignoring that the management of emergency should a whole-process one. Especially in the Big Data Era, opportunity always goes in a flash. It will become a chance if seized, but a challenge if not. Confronting with impacts of massive data, the government hasn't utilized those data to effectively serve public crisis management. The mass incident is a special type of public crisis, whose management involves social development and stability. In order to alter the mistaken ideas of the government and society, we should establish a whole-process comprehensive risk-disaster-crisis analytical framework, and use the framework in analyzing and understanding mass incidents.

1) Mass incident is not an incident actually but a dynamic process during which social risk gradually evolves in to public crisis, so that management of mass incidents should be an entire process. In terms of the logical relationship between disaster management, emergency management and risk management which is shown in Figure 2, the original disaster management should be extended to emergency management, while risk management in advance as well as latter emergency management should be added into emergency management. In other words, mass incident management should be a trinity of strategic management which necessarily includes risk management, emergency management and crisis management.

2) Mass incidents are not isolated incidents but an interactive system. There will be mutual influences among mass incidents. Demonstration effects of one mass incident will give rise to similar mass incidents, for example (Table 2): the strike of taxis in Nanjing on January 8th, 2015 led to successive taxi strikes in Changchun, Nanchang, Chengdu, Ji'nan and other places in the whole country, which have caused severe impacts on the society.

3) Subjects involved in mass incidents contain not only the government but also multiple enterprises, media, experts, stakeholders and so on. These subjects constitute a unique field operating logic.

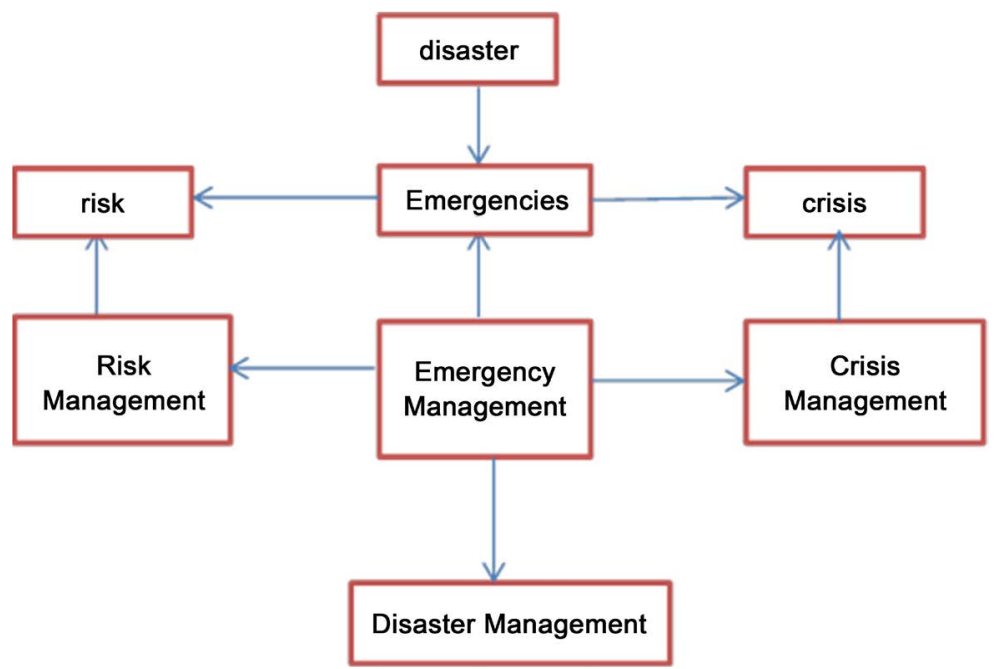

Figure 2. Logical relationships between disaster management, emergency management, risk management, and risk management. 
Table 2. Number of cities with taxi strike incidents in January 2015 and number of outages.

\begin{tabular}{ccccc}
\hline Time & Location & Event & Number of outages & Reason \\
\hline January 4, 2015 & Shenyang & Taxi strike & 1000 & Dissatisfied with drops car \\
January 8, 2015 & Nanjing & Taxi strike & Hundreds of vehicles & Too much rent \\
January 12, 2015 & Changchun & Taxi strike & Hundreds of vehicles & Too much rent \\
January 13, 2015 & Nanchang & Taxi strike & 500 & Too much rent \\
January 13, 2015 & Chengdu & Taxi strike & Thousands of vehicles & Too much rent \\
January 13, 2015 & Jinan & Taxi strike & Hundreds of vehicles & Too much rent \\
\hline
\end{tabular}

\subsection{Analytical Model of Risk Field Operation of Mass Incidents}

From the analytical point of view, we can regard the relationship between each position as an objective net or a framework, and that's the definition of field made by Bourdieu in his book The Logic of Practice. Tong Xing et al. proposed a comprehensive conceptual framework of risk field in their book Theory of Emergency Management in China: Theories, Cases and Policies, defining the unique operating field of incubation, outbreak and development of various emergencies as the risk field. Risk field is composed of risks, subjects and relevant available resources.

We can construct a mass-incident risk field from the perspectives of risks, subjects, available resources, accelerant and safety barrier.

1) Risks. Risks can bring about uncertainty of property and personnel losses. In this paper, risks in mass incidents mainly refer to labor conflicts, environmental pollution, urban housing demolition, medical disputes and so on.

2) Subjects. The subjects are actors involved, including the government, media, stakeholders, the public and others. They are core factors in the risk field, and will usually exert impacts on risk filed as well as influence its operation according to their available resources.

3) Available resources. They are usable and applicable resources in risk field, which can be employed by actors to protect their interests. Such resources primarily include economic resources, political resources and social resources. When resources obtained by an individual, a group or an organization get an edge, they will gain dominance in the risk field.

4) Accelerant. It can be used to trigger mass incidents and promote operation of risk field. The accelerant is the event or condition demanded by risk to develop into an incident.

5) Safety barrier. Physical and nonphysical methods are designed to prevent, control, or mitigate contingencies or accidents. The barrier can be divided into preventive safety barrier and responsive safety barrier. Preventive safety barrier refers to the barrier that can prevent or reduce the probability of hazardous events, while responsive safety barrier refers to barrier that can prevent or diminish the consequences of hazardous events.

In terms of the comprehensive risk-disaster-crisis analytical framework, modern 
management of mass incidents should be the organic combination of risk management, emergency management and crisis management. Therefore, we divide the risk field into three phases: the incubation phase of risk field, the outbreak phase (operating phase) of risk field and the recession phase of risk field, so as to establish mass-incident analytical model as shown in Figure 3:

1) The incubation phase of risk field. During this period, the risk and various stakeholders have been primarily formed; enterprises may carry out a risk game with the government. They are likely to shirk responsibility, such as the strike in Galanz: the enterprise kept oppressing their employees and refused to give promised salary to them.

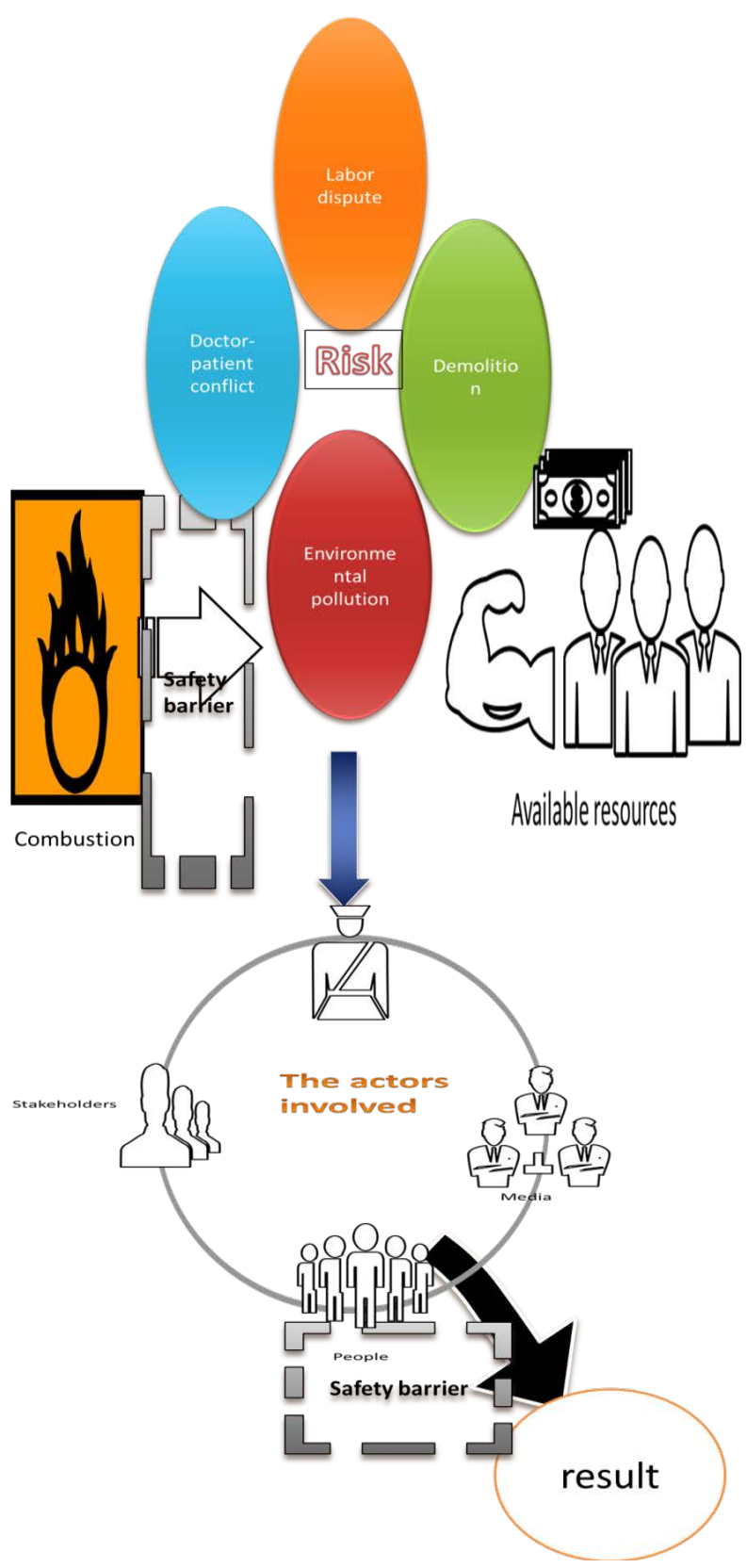

Figure 3. Group event risk field operation model. 
However, due to the absence of accelerant, risk field didn't run during the period. It should be noted that an effective safety barrier may prevent or delay the operation of risk field.

2) The operating phase of risk field. The emergence of accelerant, such as the strike and smashing incidents in Galanz: the enterprise kept oppressing their employees and refused to give promised salary to them. However, due to the absence of accelerant-several new employees drank alcohol and clamored in their dorm and consequently affected rest of other employees, which boosted the occurrence of mass incidents and promoted operation of risk field.

3) The recession phase of risk field. All acting subjects in the risk field employ their resources to compete for the right to speak, expecting favorable results through operation of field. Before outputting the results, responsive safety barrier can avoid or reduce risks of hazardous incidents. Output results may lead to a recession of risk field, or generation of new risks and thus formation of a new risk field.

\section{Case Study Based on the Mass-Incident Risk Field Operating Model}

Since the reform and opening-up, Guangdong Province has achieved obvious economic growth, whose gross domestic product has been among the forefront in the whole country. As shown in Table 3, the total GDP in Guangdong Province has ranked first among all provinces for three consecutive years from 2012 to 2014; in 2014, per capita GDP in the province exceeded ten thousand dollars, which marks that the overall development of Guangdong economic society has reached the level of moderately developed countries. However, in Guangdong Province, the development gap was prominent, the gap between rich and poor expanded, conflicts between classes and mass incidents in the society occurred frequently, which have altogether influenced the stability and development of Guangdong. According to existing information, mass incidents caused by labor conflicts have accounted for over 30\% of mass incidents involving more than a hundred people. In mass labor conflicts, the relationship between all subjects is complex. Poor treatment of such incidents will lead to the proliferation and deterioration of mass incidents, so that mass incidents cannot be effectively resolved. Therefore, confronting with such a severe situation, we should attach great significance to analyzing labor conflicts in Guangdong Province by utilizing the mass-incident risk field operating model.

On January 4th, 2016, the first weekday in 2016, due to intolerance of high monthly

Table 3. GDP of Guangdong province and the ranking table in China.

\begin{tabular}{cccc}
\hline Province & Years & Billion & GDP total ranking \\
\hline \multirow{3}{*}{ Guangdong } & 2012 & 57,068 & 1 \\
& 2013 & 62,614 & 1 \\
& 2014 & 67,792 & 1 \\
\hline
\end{tabular}

Data sources: Minnan network http://www.mnw.cn/news/china/849736.html. 
rent as well as chauffeur-driven cars and Didi's occupation of market, taxi drivers in the whole Shenzhen City went on a strike, which then brought travelling difficulties to citizens as well as traffic congestion, and especially engendered serious inconvenience to traffic hubs such as Shenzhen airport, ports of exit and entry and railway stations.

A few remarks spread in the chatting groups of taxi drivers in social network platforms like QQ and We Chat seem to become the trigger of this incident: all drivers, our business has become increasingly worse since October in 2014. We have to work 10 - 12 hours per day with only over 100 yuan of income. We must support our families and earn a living. In contrast, unlicensed chauffeur-driven unlicensed cars can be found everywhere. Relevant government departments just sit by ignoring, and we cannot take drastic action. We cannot create a disturbance, make a demonstration, and trouble the police, but we have to arouse the attention of relevant departments. Here comes the key point: On 4th January, 2016, we should all stop our business and park our cars into those licensed parking spaces in Renmin Road and Jiefang Road until relevant departments give us a favorable response. After the incident, relevant departments like Shenzhen Municipal Transportation Committee launched the emergency plan, rapidly organized personnel to communicate with taxi companies and taxi drivers; maintained transportation order and lawfully dealt with those illegal actions, so as to prevent further expansion of the incident.

The main operative subjects involved are as follows: transportation authority, taxi drivers, and taxi companies. The risks include: high monthly rent, market seizing by chauffeur-driven cars and Didi. The accelerant lies in a few remarks spread in those chatting groups of taxi drivers. On such a basis, the paper will then conduct an analysis of this mass labor conflict by utilizing the mass incident model.

1) The incubation phase of risk field. During this period, taxi drivers felt monthly rent of over 10,000 yuan was too much while their earnings became less and less. Moreover, as chauffeur-driven car-on-demand platforms such as Didi have nearly seized the market, taxis' business becomes worse, and therefore taxi drivers become increasingly dissatisfied. However, the accelerant did not occur and risk field wasn't running during such a period.

2) The operating phase of risk field. As an accelerant, the dissemination of a few remarks from online chatting groups of taxi drivers has promoted field operation. Actors involved have influenced each other by using their available resources, for examples: the Traffic Management Bureau promised to give 1000 yuan of monthly subsidy to each taxi driver and to introduce relevant policies; negotiations between taxi drivers, taxi companies and the government.

3) The recession phase of risk field. For the incident, risks haven't completely reduced, since the government has only given 1000 yuan subsidy to each taxi driver, rather than introduce relevant effective policies and management model to improve the taxi industry. Taxi companies have bought the right of use of taxi licenses usually with high costs. In order to collect the costs as soon as possible, they must transfer costs to taxi drivers, which has then given rise to the high rental of taxis. Therefore, risks still 
exist.

To analyze this mass stoppage of taxi drivers based on the mass risk field operating model, I think Shenzhen government should make improvements from the following aspects:

1) Optimization of taxi management system. The root cause of this incident lies in the outdated taxi management system, and industrial monopoly which was due to forcible maintenance of personal interests by vested interest groups and government departments. The government should spend some money redeeming license plates, accelerate the reform of market access mechanism, optimize and create a fair market, and realize marketization of the taxi industry.

2) Introduction of management regulation of chauffeur-driven cars. Unlicensed vehicles have unfairly competed with licensed vehicles, which may then exert negative influences on the order of taxi market. According to problems like the tremendous impacts confronting traditional taxi industry and lack of legal regulations in network chauffeur-driven vehicle market, Shenzhen government should introduce relevant regulations as soon as possible. It is suggested to revise the Regulations for Taxis in Shenzhen Special Economic Zone in order to effectively manage chauffeur-driven cars.

3) Accomplishment of risk assessment. The government should play the role of a regulator and supervisor, support the establishment of service evaluation system, and actively build up a communication and complaint mechanism for taxi drivers, enterprises and government, such as setting up We Chat and QQ platforms. In the era of Big Data, we should integrate data, monitor online public opinions and strengthen prediction of risks, so as to establish a sound preventive safety barrier, reduce and prevent occurrence probability of such mass incidents.

\section{Optimization Strategies of Mass Incident Governance}

In the field of emergency management, it is believed that emergency management includes management of not only crises caused by public conflicts but also crises caused by natural disasters, accidents and public health incidents. Under the context of the dual-transition period of China's society, conflicts of interests and disputes in the course of social transformation are becoming increasingly aggravated, and mass incidents occur quite frequently. Therefore, effective management of mass incidents is of great practical significance. According to the characteristics and types of mass incidents, mass-incident risk field operating model constructed on the basis of comprehensive risk-disaster-crisis framework as well as case studies, this paper has provided some governance strategies of mass incidents.

1) Adhere to a "trinity" of governance philosophy. The "trinity" of governance philosophy indicates that in governing mass incidents, we should possess the idea of combination of risk management, emergency management and crisis management. In other words, we must have the global view in governing mass incidents, and thus take the entire life cycle of incidents into account.

2) Attach importance to the role of safety barrier. We can discern the significance of 
safety barrier from the constructed mass risk field model. Safety barrier can be divided into preventive safety barrier and responsive safety barrier, so that safety barrier cannot be ignored in governing mass incidents. We should strive to establish more effective safety barriers especially preventive ones, so as to reduce or even prevent occurrence of incidents.

3) Formulate targeted governance methods according to different types of mass incidents. The representations of mass incidents triggered by contradictions among the people mainly include: labor disputes, urban housing demolition, environmental pollution and others. Based on different representations, specific issues require concrete analysis and formulation of different governance methods.

4) Application of big data technology and conduct effective management of mass incidents. The highest standard for emergency management lies in making risks disappear, or to say, take preventive measures. From the mass-incident risk operating model, we can find that the source of the entire mass incident rests with risks. Consequently, effective management and control of risks can reduce the probability of incidents and control them from the source. Therefore, in the Big Data Era, it is quite important to analyze the nature and characteristics of all mass incidents as well as to summarize risk points of various mass incidents.

\section{Conclusions}

Currently, China has been undergoing a critical period of reform and opening up. Along with progression of reform and opening up, social relations are becoming increasingly complicated, while social risks are also increasing on a daily basis. Emergence of the Big Data Era and popularization of the Internet have further expanded such risks, which thus lead to intensified social conflicts as well as upsurge of mass conflicts. Under the idea of whole-process comprehensive risk-disaster-crisis analytical framework, this paper has constructed an analysis model of mass risk field and provided some new insights for analysis of mass incidents.

On such a basis, we must master a "trinity" of governance philosophy, lay emphasis on the significance of beforehand prevention, attach importance to the functions of safety barrier and reduce impacts of the incidents. Governance must be specific, and different contingency plans and disposal methods should be made in terms of different mass incidents, which can therefore improve the ability to control mass incidents.

\section{References}

[1] Giddens, A. (1990) The Consequences of Modernity. Stanford University, Press, Stanford, 125

[2] Xiao, Y. (2012) Risk Society and China. Exploration and Controversy, No. 4, 46-51.

[3] Yuan, F. (2013) Social Risk and Social Risk Management. Economic Science Press, Beijing.

[4] Tong, X. (2012) China's Emergency Management: Theory, Practice, and Policy. Social 1st Edition, Science Literature Publishing House, Beijing, 63.

[5] Tong, X. (2012) China’s Emergency Management: Theory, Practice, and Policy. 1st Edition, 
Social Science Literature Publishing House, Beijing, 107.

[6] Yan, D.C. (2013) Network Public Opinions in Mass Events 1st Edition, . Xinhua Publishing House, Beijing, 23.

[7] Liu, B. (2013) Self-Organization Theory Based on the Evolution of Collective Labor Conflict and the Countermeasures. Capital University of Economics and Business, Beijing.

[8] Wang, H. (2010) Discussion on the Group Incidents and the Governance Path in China from the Perspective of Game Theory. Chinese Journal of Public Administration, 1, 75-77.

[9] Wang, D. (2010) Characteristics and Handling Principles of Mass Events. Chinese Journal of Public Administration, 7, 70-71.

[10] Yu, J.R. (2009) Major Types and Basic Characteristics of Mass Incidents in China. Journal of China University of Political Science and Law, 61, 114-120, 160 (in Chinese with Abstract in English).

[11] Jin, J.H., Gao, X.P., et al. (2009) Chinese Group Emergencies. 1st Edition, Beijing, 7-11.

[12] Du, Q.S. (2010) Research on the Causes and Countermeasures of Mass Events in Social Transformation. 1st Edition, Xi'an, 20-30.

[13] Wei, T.X. (2010) Discussion of Public Interest Expression and Mass Incidents. Modern Trade and Industry, 2010.

[14] Han, X.-F. (2011) Study on the Countermeasures of Hannover Enterprises after the Honda Strike in South China Sea. China Human Resource Development, 100-103.

[15] Huang, M.Z. (2012) On the Improvement of Third Party Mediation Mechanism of Medical Disputes in China. Shanghai Jiao Tong University, Shanghai.

[16] Han, J.H. (2013) Study on Enterprise Group Incident Response in Social Transformation Period. Wuhan University of Technology, Wuhan.

\section{Submit or recommend next manuscript to SCIRP and we will provide best service for you:}

Accepting pre-submission inquiries through Email, Facebook, LinkedIn, Twitter, etc.

A wide selection of journals (inclusive of 9 subjects, more than 200 journals)

Providing 24-hour high-quality service

User-friendly online submission system

Fair and swift peer-review system

Efficient typesetting and proofreading procedure

Display of the result of downloads and visits, as well as the number of cited articles

Maximum dissemination of your research work

Submit your manuscript at: http://papersubmission.scirp.org/

Or contact jss@scirp.org 